\title{
Master Fields, Drift and Dispersion in the Stochastic Limit of Quantum Theory
}

\author{
L. Accardi (accardi@volterra.mat.uniroma2.it) \\ Centro Vito Volterra. Università degli Studi di Roma "Tor Vergata". \\ 00133, Rome, Italy. \\ F.G. Cubillo (fgcubill@am.uva.es)* \\ Departamento de Análisis Matemático. Universidad de Valladolid. \\ 47005, Valladolid, Spain.
}

\begin{abstract}
This work is a detailed study of the convergence of the rescaled creation and annihilation densities, which lead to the master fields, and the form of the drift in the stochastic limit of quantum theory. The approach, based on the distributional theory of Fourier transforms, dispenses with the "analytical condition" and other restrictions usually considered and also establishes the dependence of the stochastic golden rules upon the dispersion function of the quantum field.
\end{abstract}

Keywords: Quantum open system, stochastic limit, stochastic golden rule, master field, drift, dispersion.

MSC2000: 82C10, 81S25, 81T99.

\section{Introduction}

The stochastic golden rules $[1,2]$, which arise in the stochastic limit of quantum theory as natural generalizations of the Fermi golden rule, provide a natural tool to associate a stochastic flow to any discrete system interacting with a quantum field. In the limit the field looks like a very chaotic object: a quantum white noise, i.e a $\delta$-correlated (in time) quantum field also called master field. The new evolution is an approximation of the original one which preserves much nontrivial information on the original complex system related to its decay and shift properties.

In this work we study, from an analytical point of view, the convergence of the rescaled creation and annihilation densities, which lead to the master fields, and the form of the drift term of the stochastic Schrödinger equation obtained in such limit, which contains the quantum mechanical fluctuation-dissipation relations. This approach permit us to dispense with the analytical condition and other restrictions usually considered -see Section 2- and also to establish the dependence

\footnotetext{
* Partially supported by Centro Vito Volterra (Italy), JCyL-project VA013C05 (Castilla y León) and MEC-project FIS2005-03989 (Spain).
}

(C) 2007 Kluwer Academic Publishers. Printed in the Netherlands. 
of the stochastic golden rules on certain properties of the dispersion function of the quantum field. To be precise, we shall see that, for the region where the dispersion function is regular and not constant, say $\Gamma_{1}$, every Bohr frequency of the system in its range gives rise to an independent master field, which is a quantum white noise concentrated on the corresponding resonant surface, whereas both the rest of Bohr frequencies and the open regions where the dispersion function is constant, say $\Gamma_{\alpha_{j}}$, give rise to zero master fields, except for the resonant case, see Theorem 1. In a similar way we will show that the regions $\Gamma_{\alpha_{j}}$ do not contribute to the drift term whenever the resonant case is not present, whereas for the region $\Gamma_{1}$ we obtain the usual expression with an expected additional factor, see Theorem 3 . The contribution of the singular regions of dispersion varies in each case and is not completely understood yet, see Proposition 2 and Remark 7.

The paper is organized as follows. The basic facts about the stochastic limit of quantum theory are introduced in Section 2. Section 3 describes the type of dispersion functions under study. Section 4 contains the results concerning the convergence of the rescaled creation and annihilation densities leading the master fields. The drift term of the normally ordered white noise Schrödinger equation derived from the stochastic limit is obtained in Section 5. Proofs and technical remarks are collected in Section 6. An Appendix at the end includes some of the conventions and results used in the paper.

\section{Preliminaries}

In what follows we shall consider quantum systems describing the interaction of a discrete spectrum system $S$ with free Hamiltonian

$$
H_{S}:=\sum_{r} \varepsilon_{r} P_{\varepsilon_{r}}
$$

and Bohr frequencies $\omega=\varepsilon_{r}-\varepsilon_{r^{\prime}},\left(\varepsilon_{r}, \varepsilon_{r^{\prime}} \in\right.$ Spec $\left.H_{S}\right)$, and a bosonic quantum field as reservoir $R$ with free Hamiltonian (on Fock space)

$$
H_{R}:=\int d k \omega(k) a^{+}(k) a(k),
$$

where $\omega(k)$ is the dispersion function, $a^{ \pm}(k)$ are the creation and annihilation densities, and the reference vector is mean zero Gaussian and gauge invariant, with covariance of the form

$$
\left\langle\left(\begin{array}{cc}
a^{+}(k) a\left(k^{\prime}\right) & 0 \\
0 & a\left(k^{\prime}\right) a^{+}(k)
\end{array}\right)\right\rangle=\left(\begin{array}{cc}
N(k) & 0 \\
0 & N(k)+1
\end{array}\right) \delta\left(k-k^{\prime}\right) .
$$


We will assume that the total Hamiltonian has the form

$$
H^{(\lambda)}:=H_{0}+\lambda H_{I}=H_{S}+H_{R}+\lambda H_{I},
$$

where $\lambda$ is a real coupling parameter and the interaction Hamiltonian $H_{I}$ is of dipole type, i.e. ${ }^{1}$

$$
H_{I}=\sum_{j}\left(D_{j}^{*} \otimes A\left(g_{j}\right)+D_{j} \otimes A^{*}\left(g_{j}\right)\right),
$$

where $D_{j}$ are system operators and

$$
A^{*}\left(g_{j}\right):=\int d k g_{j}(k) a^{+}(k), \quad A\left(g_{j}\right):=\int d k g^{*}(k) a(k),
$$

the functions $g_{j}$ being the cutoff or form factors. Often we will simplify the notations by omitting the symbol $\otimes$.

In the stochastic limit approach we consider the time rescaling $t \rightarrow$ $t / \lambda^{2}$ in the solution $U_{t}^{(\lambda)}=e^{i t H_{0}} e^{-i t H^{(\lambda)}}$ of the Schrödinger equation in interaction picture:

$$
\frac{\partial}{\partial t} U_{t}^{(\lambda)}=-i \lambda H_{I}(t) U_{t}^{(\lambda)}, \quad H_{I}(t)=e^{i t H_{0}} H_{I} e^{-i t H_{0}},
$$

and study the limits, in a topology to be specified, of the rescaled interaction Hamiltonian and of the rescaled propagator:

$$
\lim _{\lambda \rightarrow 0} \frac{1}{\lambda} H_{I}\left(\frac{t}{\lambda^{2}}\right)=: h_{t}, \quad \lim _{\lambda \rightarrow 0} U_{t / \lambda^{2}}^{(\lambda)}=: U_{t} .
$$

In canonical form this reduces to find the limit of the rescaled creation and annihilation densities

$$
a_{\lambda, \omega}^{ \pm}(t, k):=\frac{1}{\lambda} e^{\mp i \frac{t}{\lambda^{2}}(\omega(k)-\omega)} a^{ \pm}(k),
$$

obtaining the white noise Schrödinger equation $\frac{\partial}{\partial t} U_{t}=-i h_{t} U_{t}$, whose normally ordered form is the quantum stochastic differential equation

$$
d U_{t}=(-i d H(t)-G d t) U_{t},
$$

where $d H(t)$ is called the martingale term and

$$
G d t:=\lim _{\lambda \rightarrow 0} \frac{1}{\lambda^{2}} \int_{t}^{t+d t} d t_{1} \int_{t}^{t_{1}} d t_{2}\left\langle H_{I}\left(\frac{t_{1}}{\lambda^{2}}\right) H_{I}\left(\frac{t_{2}}{\lambda^{2}}\right)\right\rangle
$$

is known as the drift term.

Among the assumptions to achieve this program it is usual to consider the following ones:

\footnotetext{
1 The asterisk ${ }^{*}$ denotes the Hermitian conjugate for operators and the complex conjugate for scalars. For distributional densities we use the symbol ${ }^{+}$instead ${ }^{*}$.
} 
- the cut-off functions $g_{j}$ are Schwartz functions;

- the dispersion function $\omega(k)$ and the cut-off functions $g_{j}$ are related by the following analytical condition:

$$
\int_{\mathbb{R}} d t\left|\left\langle g_{i}, e^{i t \omega(p)} g_{j}\right\rangle\right|=\int_{\mathbb{R}} d t\left|\int_{\mathbb{R}^{d}} d k e^{i t \omega(k)} g_{i}^{*}(k) g_{j}(k)\right|<+\infty ;
$$

- the $(d-1)$-dimensional Lebesgue measure of the surface $\{k$ : $\omega(k)=0\}$ is equal to zero (this implies, in particular $\delta(\omega(k))=0$ ).

The techniques applied in this work, based on the distributional theory of Fourier transforms $[3,4,5]$, permit us to dispense with the above conditions and to establish the dependence of the stochastic golden rules on certain properties of the dispersion function $\omega(k)$.

\section{The Dispersion Function}

In what follows we shall assume that the dispersion function $\mathbb{R}^{d} \ni k \mapsto$ $\omega(k) \in \mathbb{R}$ is such that $\omega(k) \geq 0$ for all $k \in \mathbb{R}^{d}$ and we can write

$$
\mathbb{R}^{d}=\Gamma_{1} \cup \Gamma_{2} \cup \Gamma_{3},
$$

where:

(i) $\Gamma_{1}$ is an open set of $\mathbb{R}^{d}$ in which $\omega(k)$ is a $C^{\infty}$-function and $\nabla \omega(k) \neq 0$ for every $k \in \Gamma_{1}$. We shall denote by $\Gamma_{1}^{1}$ the range of the restriction of $\omega(k)$ to $\Gamma_{1}$, i.e.

$$
\Gamma_{1}^{1}:=\operatorname{Rang}\left(\omega_{\mid \Gamma_{1}}\right),
$$

and assume that the boundary $\partial \Gamma_{1}^{1}$ of $\Gamma_{1}^{1}$ has Lebesgue measure zero.

(ii) $\Gamma_{2}=\cup \Gamma_{\alpha_{j}}$, being $\Gamma_{\alpha_{j}}$ an open subset of $\mathbb{R}^{d}$ where the dispersion function $\omega(k)$ is constant and equal to $\alpha_{j}$, i.e.

$$
\omega(k)=\alpha_{j}, \quad \forall k \in \Gamma_{\alpha_{j}} .
$$

(iii) $\Gamma_{3}=\mathbb{R}^{d} \backslash\left(\Gamma_{1} \cup \Gamma_{2}\right)$, that is $\Gamma_{3}$ contains the boundaries of $\Gamma_{1}$ and $\Gamma_{2}$ and other possible regions of singular points of the dispersion function $\omega(k)$. 


\section{Convergence of the Rescaled Densities}

Let us study the convergence, in the sense of correlators, of the rescaled creation and annihilation densities given in Eq.(2). To simplify the notation we restrict our attention to the vacuum reference vector, so that $N(k)=0$ (see Eq.(1)). The extension of the results to the general case is immediate. Moreover, because of the mean zero Gaussianity, we have only to prove the convergence, in the sense of Schwartz distributions [4], of the covariance

$$
\left\langle a_{\lambda, \omega}(t, k) a_{\lambda, \omega^{\prime}}^{+}\left(t^{\prime}, k^{\prime}\right)\right\rangle=\frac{1}{\lambda^{2}} e^{-i \frac{t-t^{\prime}}{\lambda^{2}}(\omega(k)-\omega)+i \frac{t^{\prime}}{\lambda^{2}}\left(\omega-\omega^{\prime}\right)} \delta\left(k-k^{\prime}\right),
$$

i.e. we must calculate, for any Schwartz test functions $\phi, \varphi, f$ and $g$,

$$
\lim _{\lambda \rightarrow 0} \int d t d t^{\prime} d k d k^{\prime} \phi(t) \varphi\left(t^{\prime}\right) f(k) g\left(k^{\prime}\right)\left\langle a_{\lambda, \omega}(t, k) a_{\lambda, \omega^{\prime}}^{+}\left(t^{\prime}, k^{\prime}\right)\right\rangle .
$$

The following theorem shows that, on $\Gamma_{1}$, every Bohr frequency $\omega$ in the open range $\Gamma_{1}^{1}$ of the dispersion function gives rise to an independent master field, which is a quantum white noise concentrated over the resonant surface $\omega(k)-\omega=0$, and the rest of Bohr frequencies give rise to zero master fields - notice the factor $\chi_{\Gamma_{1}^{1}}$, while, on the open regions $\Gamma_{\alpha_{j}}$ where the dispersion function is constant, the limit does not exist in the resonant case $\alpha_{j}=\omega=\omega^{\prime}$ and again gives rise to zero master fields otherwise.

THEOREM 1. Under the conditions for $\omega(k)$ given in Section 3, in the sense of Schwartz distributions, i.e. in $\mathcal{S}^{\prime}\left(\mathbb{R}^{2 d+2}\right)$ :

(a) Over $\Gamma_{1}$, if $\omega$ doesn't belong to the boundary $\partial \Gamma_{1}^{1}$ of $\Gamma_{1}^{1}$,

$$
\left.\lim _{\lambda \rightarrow 0}\left\langle a_{\lambda, \omega}(t, k) a_{\lambda, \omega^{\prime}}^{+}\left(t^{\prime}, k^{\prime}\right)\right\rangle\right|_{\Gamma_{1}}=\delta_{\omega, \omega^{\prime}} 2 \pi \delta\left(t-t^{\prime}\right) \delta\left(k-k^{\prime}\right) \delta(\omega(k)-\omega) \chi_{\Gamma_{1}^{1}}(\omega) .
$$

(b) Over each $\Gamma_{\alpha_{j}}$,

$$
\left.\lim _{\lambda \rightarrow 0}\left\langle a_{\lambda, \omega}(t, k) a_{\lambda, \omega^{\prime}}^{+}\left(t^{\prime}, k^{\prime}\right)\right\rangle\right|_{\Gamma_{\alpha_{j}}}= \begin{cases}\text { doesn't exist, } & \text { if } \alpha_{j}=\omega=\omega^{\prime}, \\ 0, & \text { if } \alpha_{j} \neq \omega \text { or } \alpha_{j} \neq \omega^{\prime} .\end{cases}
$$

The proof of this result (given in Section 6) casts some light on the resonant case $\alpha_{j}=\omega=\omega^{\prime}$ of item (b): Over each $\Gamma_{\alpha_{j}}$ the final expression in our calculations is ${ }^{2}$

$$
\lim _{\lambda \rightarrow 0} \frac{2 \pi}{\lambda^{2}} \phi^{\vee}\left(\frac{\alpha_{j}-\omega}{\lambda^{2}}\right) \varphi^{\wedge}\left(\frac{\alpha_{j}-\omega^{\prime}}{\lambda^{2}}\right) \int_{\Gamma_{\alpha_{j}}} d k f(k) g(k)=
$$

\footnotetext{
${ }^{2}$ See Appendix A for notation and conventions.
} 


$$
=\lim _{\lambda \rightarrow 0} \frac{2 \pi}{\lambda^{2}} \phi^{\vee}(0) \varphi^{\wedge}(0) \int_{\Gamma_{\alpha_{j}}} d k f(k) g(k),
$$

which is equal to zero when $\phi^{\vee}(0)=0$ or $\varphi^{\wedge}(0)=0$, or $\pm \infty$ otherwise. Thus, if we restrict our attention to test functions with zero mean in time, the limit also exists in this case and is equal to zero.

What happens over $\Gamma_{3}$ or when $\omega \in \partial \Gamma_{1}^{1}$ ? As Proposition 2 shows below, the answer depends on the dispersion function. Indeed, let us consider dispersion functions of the form

$$
\omega(k)=|k|^{\mu}, \quad \mu>0,
$$

for which $\Gamma_{1}=\mathbb{R}^{d} \backslash\{0\}, \Gamma_{2}=\emptyset, \Gamma_{3}=\{0\}, \Gamma_{1}^{1}=(0, \infty)$ and $\partial \Gamma_{1}^{1}=\{0\}$, so that the frequency of interest is $\omega=0$. We obtain in this case:

PROPOSITION 2. For dispersion functions of the form (5),

$$
\begin{aligned}
& \lim _{\lambda \rightarrow 0}\left\langle a_{\lambda, \omega}(t, k) a_{\lambda, \omega^{\prime}}^{+}\left(t^{\prime}, k^{\prime}\right)\right\rangle= \\
&=\left\{\begin{array}{cc}
\delta_{\omega, \omega^{\prime}} 2 \pi \delta\left(t-t^{\prime}\right) \delta\left(k-k^{\prime}\right) \delta(\omega(k)-\omega), & \text { if } \omega>0, \\
0, & \text { if } \omega<0,
\end{array}\right.
\end{aligned}
$$

and

$$
\begin{aligned}
&\left.\lim _{\lambda \rightarrow 0}\left\langle a_{\lambda, \omega}(t, k) a_{\lambda, \omega^{\prime}}^{+}\left(t^{\prime}, k^{\prime}\right)\right\rangle\right|_{\omega=0}= \\
& \quad=\left\{\begin{array}{cc}
0, & \text { if } d-\mu>0, \\
\delta_{\omega, \omega^{\prime}} \frac{2 \pi^{d / 2+1}}{\Gamma(d / 2)} \delta\left(t-t^{\prime}\right) \delta\left(k-k^{\prime}\right) \delta(k), & \text { if } d-\mu=0 .
\end{array}\right.
\end{aligned}
$$

(For $\omega=0$ and $d-\mu<0$ our techniques do not give an answer.)

\section{The Drift}

As Eq.(4) shows, the drift term Gdt in the stochastic Schrödinger equation given in Eq.(3) is the limit of the expectation value in the reservoir state of the second term in the iterated series solution for the rescaled Shrödinger equation in interaction picture.

In the following theorem we show that the open region $\Gamma_{2}$ does not contribute to the drift term whenever the resonant case $\alpha_{k}=\omega$ is not present, whereas for the region $\Gamma_{1}$ we obtain the usual expression for the drift with a $\chi_{\Gamma_{1}^{1}}$ factor added. The contribution of the singular region $\Gamma_{3}$ to the drift has not been determined yet. 
THEOREM 3. Under the conditions for $\omega(k)$ given in Section 3 we have:

(i) If $\Gamma_{2}$ is not empty and no Bohr frequency $\omega$ of the system coincides with one of the values $\alpha_{k}$, then the contribution of the region $\Gamma_{2}$ to the drift term is zero, whereas if any of the Bohr frequencies $\omega$ of the system coincides with one of the values $\alpha_{k}$, then $G$ does not exist.

(ii) Otherwise

$$
\begin{aligned}
G=\sum_{i j} \sum_{\omega} & \left(\left(g_{i} \mid g_{j}\right)_{\omega}^{-} E_{\omega}^{*}\left(D_{i}\right) E_{\omega}\left(D_{j}\right)+\left(g_{i} \mid g_{j}\right)_{\omega}^{+*} E_{\omega}\left(D_{i}\right) E_{\omega}^{*}\left(D_{j}\right)+\right. \\
& \left.+ \text { The part corresponding to the singular region } \Gamma_{3}\right)
\end{aligned}
$$

where, for each Bohr frequency $\omega$, the $E_{\omega}\left(D_{j}\right)$ are system operators defined by

$$
\begin{gathered}
E_{\omega}\left(D_{j}\right):=\sum_{\varepsilon_{r} \in F_{\omega}} P_{\varepsilon_{r}-\omega} D_{j} P_{\varepsilon_{r}}, \\
F_{\omega}:=\left\{\varepsilon_{r} \in S p e c H_{S}: \varepsilon_{r}-\omega \in \operatorname{Spec} H_{S}\right\},
\end{gathered}
$$

and the explicit forms of the constants $\left(g_{i} \mid g_{j}\right)_{\omega}^{ \pm}$are

$$
\begin{gathered}
\left(g_{i} \mid g_{j}\right)_{\omega}^{-}=\pi \chi_{\Gamma_{1}^{1}}(\omega) \int_{\Gamma_{1}} d k g_{i}^{*}(k) g_{j}(k)(N(k)+1) \delta(\omega(k)-\omega)- \\
-i P . P . \int_{\Gamma_{1}} d k g_{i}^{*}(k) g_{j}(k) \frac{(N(k)+1)}{\omega(k)-\omega} \\
\left(g_{i} \mid g_{j}\right)_{\omega}^{+}=\pi \chi_{\Gamma_{1}^{1}}(\omega) \int_{\Gamma_{1}} d k g_{i}^{*}(k) g_{j}(k) N(k) \delta(\omega(k)-\omega)- \\
-i P . P . \int_{\Gamma_{1}} d k g_{i}^{*}(k) g_{j}(k) \frac{N(k)}{\omega(k)-\omega} .
\end{gathered}
$$

The constants $\left(g_{i} \mid g_{j}\right)_{\omega}^{ \pm}$, known as generalized susceptivities, contain all the physical information on the original Hamiltonian system and can be considered as the prototype of quantum mechanical fluctuationdissipation relations.

\section{Proofs and Remarks}

Proof of Theorem 1: For any test functions $\phi, \varphi, f$ and $g$, we must calculate

$$
\begin{aligned}
I:= & \lim _{\lambda \rightarrow 0} \int d t d t^{\prime} d k d k^{\prime} \phi(t) \varphi\left(t^{\prime}\right) f(k) g\left(k^{\prime}\right)\left\langle a_{\lambda, \omega}(t, k) a_{\lambda, \omega^{\prime}}^{+}\left(t^{\prime}, k^{\prime}\right)\right\rangle= \\
= & \lim _{\lambda \rightarrow 0} \int d t d t^{\prime} d k d k^{\prime} \phi(t) \varphi\left(t^{\prime}\right) f(k) g\left(k^{\prime}\right) \times \\
& \times \frac{1}{\lambda^{2}} e^{-i \frac{t-t^{\prime}}{\lambda^{2}}(\omega(k)-\omega)+i \frac{t^{\prime}}{\lambda^{2}}\left(\omega-\omega^{\prime}\right)} \delta\left(k-k^{\prime}\right)= \\
= & \lim _{\lambda \rightarrow 0} \int d t d t^{\prime} d k \phi(t) \varphi\left(t^{\prime}\right) f(k) g(k) \frac{1}{\lambda^{2}} e^{-i \frac{t-t^{\prime}}{\lambda^{2}}(\omega(k)-\omega)+i \frac{t^{\prime}}{\lambda^{2}}\left(\omega-\omega^{\prime}\right)} .
\end{aligned}
$$


Making the change of variables $\left(t-t^{\prime}\right) / \lambda^{2}=\sigma, t^{\prime}=\tau$, we find

$$
I=\lim _{\lambda \rightarrow 0} \int d \sigma d \tau d k \phi\left(\tau+\lambda^{2} \sigma\right) \varphi(\tau) f(k) g(k) e^{i \frac{\tau}{\lambda^{2}}\left(\omega-\omega^{\prime}\right)} e^{-i \sigma(\omega(k)-\omega)} .
$$

The integrand belongs to $L^{1}\left(\mathbb{R}^{d+2}\right)$ for every $\lambda \neq 0$ and then, by Fubini's theorem, we can integrate in any order. Then, $I$ equals

$$
\lim _{\lambda \rightarrow 0} \int d \tau \varphi(\tau) e^{i \frac{\tau}{\lambda^{2}}\left(\omega-\omega^{\prime}\right)} \int d \sigma \phi\left(\tau+\lambda^{2} \sigma\right) e^{i \sigma \omega} \int d k f(k) g(k) e^{-i \sigma \omega(k)} .
$$

Now, let us put

$$
\begin{gathered}
\int d k f(k) g(k) e^{-i \sigma \omega(k)}=\int_{\Gamma_{1} \cup \Gamma_{2} \cup \Gamma_{3}} d k f(k) g(k) e^{-i \sigma \omega(k)}= \\
=\int_{\Gamma_{1}} d k f(k) g(k) e^{-i \sigma \omega(k)}+\sum_{j} \int_{\Gamma_{\alpha_{j}}} d k f(k) g(k) e^{-i \sigma \omega(k)}+ \\
+\int_{\Gamma_{3}} d k f(k) g(k) e^{-i \sigma \omega(k)}
\end{gathered}
$$

and

$$
I=I_{1}+I_{2}+I_{3},
$$

where $I_{j},(j=1,2,3)$, corresponds to the integral over $\Gamma_{j}$ with respect to $k$ on $I$.

Over $\Gamma_{1}$, using the notation given in Section 3.(i), we have (the symbol $^{\wedge}$ denotes the Fourier transform and the symbol ${ }^{\vee}$ the inverse Fourier transform, see appendix A)

$$
\begin{gathered}
\int_{\Gamma_{1}} d k f(k) g(k) e^{-i \sigma \omega(k)}=\int_{\Gamma_{1}^{1}} d u_{1} e^{i \tau u_{1}} \Omega_{f g}\left(u_{1}\right)= \\
=\int d u_{1} e^{-i \sigma u_{1}} \chi_{\Gamma_{1}^{1}}\left(u_{1}\right) \Omega_{f g}\left(u_{1}\right)=\sqrt{2 \pi}\left[\chi_{\Gamma_{1}^{1}} \Omega_{f g}\right]^{\vee}(\sigma)
\end{gathered}
$$

and get

$$
\begin{aligned}
I_{1} & =\lim _{\lambda \rightarrow 0} \sqrt{2 \pi} \int d \tau \varphi(\tau) e^{i \frac{\tau}{\lambda^{2}}\left(\omega-\omega^{\prime}\right)} \int d \sigma \phi\left(\tau+\lambda^{2} \sigma\right)\left[\chi_{\Gamma_{1}^{1}} \Omega_{f g}\right]^{\vee}(\sigma) e^{i \sigma \omega}= \\
& =\lim _{\lambda \rightarrow 0} 2 \pi \int d \tau \varphi(\tau) e^{i \frac{\tau}{\lambda^{2}}\left(\omega-\omega^{\prime}\right)}\left[\phi\left(\tau+\lambda^{2} \sigma\right)\left[\chi_{\Gamma_{1}^{1}} \Omega_{f g}\right]^{\vee}(\sigma)\right]^{\wedge}(\omega) .
\end{aligned}
$$

Since $\chi_{\Gamma_{1}^{1}} \Omega_{f g}$ satisfies the conditions of Jordan's test (Theorem 9) or Dini's test (Theorem 10) for every interior point of $\Gamma_{1}^{1}$ and is equal to zero outside of $\Gamma_{1}^{1}$, we have

$$
\left[\chi_{\Gamma_{1}^{1}} \Omega_{f g}\right]^{\vee \wedge}(\omega)=\left[\chi_{\Gamma_{1}^{1}} \Omega_{f g}\right](\omega), \quad \omega \in \mathbb{R} \backslash \partial \Gamma_{1}^{1} .
$$


On the other hand,

$$
\left[\phi\left(\tau+\lambda^{2} \sigma\right)\right]^{\wedge}(\omega)=\frac{1}{\lambda^{2}} \phi^{\wedge}\left(\frac{\omega}{\lambda^{2}}\right) e^{-i \tau \frac{\omega}{\lambda^{2}}} .
$$

Therefore, using the fact that the Fourier transform of a product is equal to the convolution of the Fourier transforms of the factors, from Eq.(9) we obtain

$I_{1}=\lim _{\lambda \rightarrow 0} \frac{2 \pi}{\lambda^{2}} \int d \tau \varphi(\tau) e^{i \frac{\tau}{\lambda^{2}}\left(\omega-\omega^{\prime}\right)} \int d t \phi^{\wedge}\left(\frac{t}{\lambda^{2}}\right) e^{-i \tau \frac{t}{\lambda^{2}}}\left[\chi_{\Gamma_{1}^{1}} \Omega_{f g}\right](\omega-t)$, since $\partial \Gamma_{1}^{1}$ has Lebesgue measure zero.

Now, interchanging the order of integration $\left(\phi \chi_{\Gamma_{1}^{1}} \Omega_{f g}\right.$ belongs to $L^{1}(\mathbb{R})$ for every test function $\phi$ ),

$$
\begin{aligned}
I_{1} & =\lim _{\lambda \rightarrow 0} \frac{2 \pi}{\lambda^{2}} \int d t \phi^{\wedge}\left(\frac{t}{\lambda^{2}}\right)\left[\chi_{\Gamma_{1}^{1}} \Omega_{f g}\right](\omega-t) \int d \tau \varphi(\tau) e^{i \frac{\tau}{\lambda^{2}}\left(\omega-\omega^{\prime}-t\right)}= \\
& =\lim _{\lambda \rightarrow 0} \frac{(2 \pi)^{3 / 2}}{\lambda^{2}} \int d t \phi^{\wedge}\left(\frac{t}{\lambda^{2}}\right)\left[\chi_{\Gamma_{1}^{1}} \Omega_{f g}\right](\omega-t) \varphi^{\wedge}\left(\frac{\omega-\omega^{\prime}-t}{\lambda^{2}}\right)=
\end{aligned}
$$

(by the commutativity of the convolution)

$$
=\lim _{\lambda \rightarrow 0} \frac{(2 \pi)^{3 / 2}}{\lambda^{2}} \int d t \phi^{\wedge}\left(\frac{\omega-t}{\lambda^{2}}\right)\left[\chi_{\Gamma_{1}^{1}} \Omega_{f g}\right](t) \varphi^{\wedge}\left(\frac{t-\omega^{\prime}}{\lambda^{2}}\right)=
$$

(taking the change of variable $t=\lambda^{2} \sigma$ )

$$
=\lim _{\lambda \rightarrow 0}(2 \pi)^{3 / 2} \int d \sigma \phi^{\wedge}\left(\frac{\omega}{\lambda^{2}}-\sigma\right)\left[\chi_{\Gamma_{1}^{1}} \Omega_{f g}\right]\left(\lambda^{2} \sigma\right) \varphi^{\wedge}\left(\sigma-\frac{\omega^{\prime}}{\lambda^{2}}\right)=
$$

(taking the change of variable $\sigma-\frac{\omega^{\prime}}{\lambda^{2}}=\tau$ )

$$
=\lim _{\lambda \rightarrow 0}(2 \pi)^{3 / 2} \int d \tau \phi^{\wedge}\left(\frac{\omega-\omega^{\prime}}{\lambda^{2}}-\tau\right)\left[\chi_{\Gamma_{1}^{1}} \Omega_{f g}\right]\left(\lambda^{2} \tau+\omega^{\prime}\right) \varphi^{\wedge}(\tau) .
$$

Since $\chi_{\Gamma_{1}^{1}} \Omega_{f g}$ is a bounded function, we can apply the dominated convergence theorem and get:

(1) If $\omega=\omega^{\prime} \in \mathbb{R} \backslash \partial \Gamma_{1}^{1}$, since $\chi_{\Gamma_{1}^{1}} \Omega_{f g}$ is continuous at $\omega$ and $\phi, \varphi \in$ $\mathcal{S}(\mathbb{R})$,

$$
\begin{aligned}
I_{1} & =(2 \pi)^{3 / 2}\left[\chi_{\Gamma_{1}^{1}} \Omega_{f g}\right](\omega) \int d \tau \phi^{\wedge}(-\tau) \varphi^{\wedge}(\tau)= \\
& =(2 \pi)^{3 / 2}\left[\chi_{\Gamma_{1}^{1}} \Omega_{f g}\right](\omega)\left[\phi^{\wedge} * \varphi^{\wedge}\right](0)= \\
& =(2 \pi)^{3 / 2}\left[\chi_{\Gamma_{1}^{1}} \Omega_{f g}\right](\omega)[\phi \varphi]^{\wedge}(0)= \\
& =(2 \pi)^{3 / 2}\left[\chi_{\Gamma_{1}^{1}} \Omega_{f g}\right](\omega) \frac{1}{\sqrt{2 \pi}} \int d t \phi(t) \varphi(t)= \\
& =\left\langle 2 \pi \delta\left(t-t^{\prime}\right) \delta\left(k-k^{\prime}\right) \delta(\omega(k)-\omega) \chi_{\Gamma_{1}^{1}}(\omega), f(k) g\left(k^{\prime}\right) \phi(t) \varphi\left(t^{\prime}\right)\right\rangle .
\end{aligned}
$$


(2) If $\omega \neq \omega^{\prime} \in \mathbb{R} \backslash \partial \Gamma_{1}^{1}$, by Riemann-Legesgue theorem,

$$
\lim _{\lambda \rightarrow 0} \phi^{\wedge}\left(\frac{\omega-\omega^{\prime}}{\lambda^{2}}-\tau\right)=0
$$

and $I_{1}=0$.

On the other hand, over $\Gamma_{2}$ we have

$$
\begin{aligned}
& I_{2}=\lim _{\lambda \rightarrow 0} \int d \tau \varphi(\tau) e^{i \frac{\tau}{\lambda^{2}}\left(\omega-\omega^{\prime}\right)} \int d \sigma \phi\left(\tau+\lambda^{2} \sigma\right) e^{i \sigma \omega} \times \\
& \times \int_{\Gamma_{2}} d k f(k) g(k) e^{-i \sigma \omega(k)}=\lim _{\lambda \rightarrow 0} \int d \tau \varphi(\tau) e^{i \frac{\tau}{\lambda^{2}}\left(\omega-\omega^{\prime}\right)} \times \\
& \times \int d \sigma \phi\left(\tau+\lambda^{2} \sigma\right) e^{i \sigma \omega} \int d k \sum_{j} \chi_{\Gamma_{\alpha_{j}}}(k) f(k) g(k) e^{-i \sigma \alpha_{j}} .
\end{aligned}
$$

Since $f, g \in \mathcal{S}\left(\mathbb{R}^{d}\right)$ and $\phi, \varphi \in \mathcal{S}(\mathbb{R})$, by the dominated convergence theorem we can interchange sum and integrals, so that $I_{2}$ equals

$\lim _{\lambda \rightarrow 0} \sum_{j} \int d \tau \varphi(\tau) e^{i \frac{\tau}{\lambda^{2}}\left(\omega-\omega^{\prime}\right)} \int d \sigma \phi\left(\tau+\lambda^{2} \sigma\right) e^{-i \sigma\left(\alpha_{j}-\omega\right)} \int_{\Gamma_{\alpha_{j}}} d k f(k) g(k)$.

Taking the change of variable $\lambda^{2} \sigma=u$,

$$
\begin{aligned}
\int d \sigma \phi\left(\tau+\lambda^{2} \sigma\right) e^{-i \sigma\left(\alpha_{j}-\omega\right)} & =\frac{1}{\lambda^{2}} \int d u \phi(\tau+u) e^{-i \frac{u}{\lambda^{2}}\left(\alpha_{j}-\omega\right)}= \\
& =\frac{\sqrt{2 \pi}}{\lambda^{2}} \phi^{\vee}\left(\frac{\alpha_{j}-\omega}{\lambda^{2}}\right) e^{i \tau \frac{\alpha_{j}-\omega}{\lambda^{2}}} .
\end{aligned}
$$

Therefore, $I_{2}$ equals

$$
\begin{gathered}
\lim _{\lambda \rightarrow 0} \sum_{j} \frac{\sqrt{2 \pi}}{\lambda^{2}} \phi^{\vee}\left(\frac{\alpha_{j}-\omega}{\lambda^{2}}\right) \int d \tau \varphi(\tau) e^{i \frac{\tau}{\lambda^{2}}\left(\alpha_{j}-\omega^{\prime}\right)} \int_{\Gamma_{\alpha_{j}}} d k f(k) g(k)= \\
=\lim _{\lambda \rightarrow 0} \sum_{j} \frac{2 \pi}{\lambda^{2}} \phi^{\vee}\left(\frac{\alpha_{j}-\omega}{\lambda^{2}}\right) \varphi^{\wedge}\left(\frac{\alpha_{j}-\omega^{\prime}}{\lambda^{2}}\right) \int_{\Gamma_{\alpha_{j}}} d k f(k) g(k) .
\end{gathered}
$$

Term by term we have:

(1) If $\alpha_{j}=\omega=\omega^{\prime}$,

$$
\begin{gathered}
\lim _{\lambda \rightarrow 0} \frac{2 \pi}{\lambda^{2}} \phi^{\vee}\left(\frac{\alpha_{j}-\omega}{\lambda^{2}}\right) \varphi^{\wedge}\left(\frac{\alpha_{j}-\omega^{\prime}}{\lambda^{2}}\right) \int_{\Gamma_{\alpha_{j}}} d k f(k) g(k)= \\
=\lim _{\lambda \rightarrow 0} \frac{2 \pi}{\lambda^{2}} \phi^{\vee}(0) \varphi^{\wedge}(0) \int_{\Gamma_{\alpha_{j}}} d k f(k) g(k),
\end{gathered}
$$

which is equal to zero when $\phi^{\vee}(0)=0$ or $\varphi^{\wedge}(0)=0$, or $\pm \infty$ otherwise. Thus, the corresponding limit in $\mathcal{S}^{\prime}\left(\mathbb{R}^{2 d+2}\right)$ doesn't exist. 
(2) If $\alpha_{j} \neq \omega$ or $\alpha_{j} \neq \omega^{\prime}$, since $\phi, \varphi \in \mathcal{S}(\mathbb{R})$, by Riemann-Lebesgue theorem,

$$
\lim _{\lambda \rightarrow 0} \frac{2 \pi}{\lambda^{2}} \phi^{\vee}\left(\frac{\alpha_{j}-\omega}{\lambda^{2}}\right) \varphi^{\wedge}\left(\frac{\alpha_{j}-\omega^{\prime}}{\lambda^{2}}\right) \int_{\Gamma_{\alpha_{j}}} d k f(k) g(k)=0 .
$$

This concludes the proof.

REMARK 4. Let us consider the expression

$$
\lim _{\lambda \rightarrow 0} \int d \sigma d \tau d k \beta_{\lambda}(\sigma, \tau, k)
$$

A Vitali theorem says that we can interchange limit and integral in (10) if for every $\epsilon>0$ there exists a compact set $K_{\epsilon} \subset \mathbb{R}^{d+2}$ such that, for all $\lambda \in\left(0, \epsilon_{0}\right)$,

(i) $\int_{K_{\epsilon}^{c}} d \sigma d \tau d k\left|\beta_{\lambda}(\sigma, \tau, k)\right|<\epsilon$,

(ii) $\left|\beta_{\lambda}(\sigma, \tau, k)\right|<c, \forall(\sigma, \tau, k) \in K_{\epsilon}$,

where $\epsilon_{0}$ and $c$ are two fixed positive constants.

Can we apply this Vitali theorem to study the expression for $I$ given in Eq.(8), i.e. when

$$
\beta_{\lambda}(\sigma, \tau, k)=\phi\left(\tau+\lambda^{2} \sigma\right) \varphi(\tau) f(k) g(k) e^{i \frac{\tau}{\lambda^{2}}\left(\omega-\omega^{\prime}\right)} e^{-i \sigma(\omega(k)-\omega)} ?
$$

In this case the condition (ii) is clearly satisfied since

$$
\left|\beta_{\lambda}(\sigma, \tau, k)\right| \leq\|\phi\|_{\infty}\|\varphi\|_{\infty}\|f g\|_{\infty}, \quad \forall(\sigma, \tau, k) \in \mathbb{R}^{d+2} .
$$

But the answer to the question is in general negative because, as regards condition (i), we can assume, without lost of generality, that

$$
K_{\epsilon}=[a, b] \times\left[a^{\prime}, b^{\prime}\right] \times \bar{B}(0, r) \subset \mathbb{R} \times \mathbb{R} \times \mathbb{R}^{d},
$$

so that

$$
\begin{gathered}
K_{\epsilon}^{c}=[a, b]^{c} \times\left[a^{\prime}, b^{\prime}\right] \times \bar{B}(0, r) \bigcup[a, b] \times\left[a^{\prime}, b^{\prime}\right]^{c} \times \bar{B}(0, r) \bigcup \\
\bigcup[a, b] \times\left[a^{\prime}, b^{\prime}\right] \times \bar{B}(0, r)^{c} \bigcup[a, b]^{c} \times\left[a^{\prime}, b^{\prime}\right]^{c} \times \bar{B}(0, r) \bigcup \\
\bigcup[a, b]^{c} \times\left[a^{\prime}, b^{\prime}\right] \times \bar{B}(0, r)^{c} \bigcup[a, b] \times\left[a^{\prime}, b^{\prime}\right]^{c} \times \bar{B}(0, r)^{c} \bigcup \\
\bigcup[a, b]^{c} \times\left[a^{\prime}, b^{\prime}\right]^{c} \times \bar{B}(0, r)^{c}
\end{gathered}
$$

and then

$$
\int_{K_{\epsilon}^{c}} d \sigma d \tau d k\left|\beta_{\lambda}(\sigma, \tau, k)\right|=
$$




$$
\begin{aligned}
& =\int_{\left([a, b] \times\left[a^{\prime}, b^{\prime}\right] \times \bar{B}(0, r)\right)^{c}} d \sigma d \tau d k\left|\phi\left(\tau+\lambda^{2} \sigma\right) \varphi(\tau) f(k) g(k)\right|= \\
= & \int_{[a, b] c \times\left[a^{\prime}, b^{\prime}\right]} d \sigma d \tau\left|\phi\left(\tau+\lambda^{2} \sigma\right) \varphi(\tau)\right| \int_{\bar{B}(0, r)} d k|f(k) g(k)|+\cdots
\end{aligned}
$$

But one cannot find $K_{\epsilon}$ such that this first integral verifies (ii) for every $\lambda \in\left(0, \epsilon_{0}\right)$.

Proof of Proposition 2: Eq.(6) is just Theorem 1.(a) for this particular case, for which we can take as new variables

$$
u_{1}=\omega(k), u_{2}=\theta_{1}, \ldots, u_{d}=\theta_{d-1},
$$

where $\theta_{1}, \ldots, \theta_{d-1}$ are the usual angles in spherical coordinates, so that

$$
\int d k f(k) g(k) e^{-i \sigma \omega(k)}=\int_{0}^{\infty} d u_{1} e^{-i \sigma u_{1}} \frac{u_{1}^{d / \mu-1}}{\mu} \int_{S_{u_{1}^{1 / \mu}}} d \sigma_{S_{1}} f(u) g(u) .
$$

being $S_{u_{1}^{1 / \mu}}$ the sphere centered at the origin with radius $u_{1}^{1 / \mu}$ and $d \sigma_{S_{1}}$ the Euclidean element of surface for the sphere $S_{1}$. That is, with the notation used in the proof of Theorem 1.(a),

$$
\chi_{\Gamma_{1}^{1}}\left(u_{1}\right) \Omega_{f g}\left(u_{1}\right)=\chi_{(0, \infty)}\left(u_{1}\right) \frac{u_{1}^{d / \mu-1}}{\mu} \int_{S_{u_{1}^{1 / \mu}}} d \sigma_{S_{1}} f(u) g(u) .
$$

What happens for $\omega=0$ ? Since

$$
\lim _{u_{1} \rightarrow 0} \int_{S_{u_{1}^{1 / \mu}}} d \sigma_{S_{1}} f(u) g(u)=f(0) g(0) \int_{S_{1}} d \sigma_{S_{1}},
$$

the function on $\chi_{\Gamma_{1}^{1}} \Omega_{f g}$ is continuous at $u_{1}=0 \mathrm{iff}$

$$
\lim _{u_{1} \rightarrow 0} u_{1}^{d / \mu-1} f(u) g(u)=\lim _{|k| \rightarrow 0}|k|^{d-\mu} f(k) g(k)=0 .
$$

This will be the case for every test functions $f, g$, iff $d-\mu>0$, so that

$$
\left[\chi_{\Gamma_{1}^{1}} \Omega_{f g}\right]^{\vee \wedge}(0)=\left[\chi_{\Gamma_{1}^{1}} \Omega_{f g}\right](0)=0 .
$$

Then, reasoning as in proof of Theorem 1.(a) we get

$$
\left.\lim _{\lambda \rightarrow 0}\left\langle a_{\lambda, \omega}(t, k) a_{\lambda, \omega}^{+}\left(t^{\prime}, k^{\prime}\right)\right\rangle\right|_{\omega=0}=0, \quad \text { if } d-\mu>0 .
$$


When $d-\mu=0$, the function $\chi_{\Gamma_{1}^{1}} \Omega_{f g}$ is not continuous at $u_{1}=0$, but the lateral limits exist and are finite: ${ }^{3}$

$$
\begin{aligned}
& \lim _{u_{1} \rightarrow 0^{-}}\left[\chi_{\Gamma_{1}^{1}} \Omega_{f g}\right]\left(u_{1}\right)=0, \\
& \lim _{u_{1} \rightarrow 0^{+}}\left[\chi_{\Gamma_{1}^{1}} \Omega_{f g}\right]\left(u_{1}\right)=f(0) g(0) \int_{S_{1}} d \sigma_{S_{1}}=f(0) g(0) \frac{2 \pi^{d / 2}}{\Gamma(d / 2)} .
\end{aligned}
$$

Then, by Jordan's test (Theorem 9$),\left[\chi_{\Gamma_{1}^{1}} \Omega_{f g}\right]^{\vee \wedge}(0)$ is the mean value of the two lateral limits, so that

$$
\left.\lim _{\lambda \rightarrow 0}\left\langle a_{\lambda, \omega}(t, k) a_{\lambda, \omega}^{+}\left(t^{\prime}, k^{\prime}\right)\right\rangle\right|_{\omega=0}=\frac{2 \pi^{d / 2+1}}{\Gamma(d / 2)} \delta_{\omega, \omega^{\prime}} \delta\left(t-t^{\prime}\right) \delta\left(k-k^{\prime}\right) \delta(k),
$$

if $d-\mu=0$.

Proof of Theorem 3: Let us introduce the set of energy differences (Bohr frequencies)

$$
F:=\left\{\omega=\varepsilon_{r}-\varepsilon_{r^{\prime}}: \varepsilon_{r}, \varepsilon_{r^{\prime}} \in \operatorname{Spec} H_{S}\right\}
$$

and, for each $\omega \in F$, the set

$$
\begin{aligned}
F_{\omega} & :=\left\{\varepsilon_{r} \in \operatorname{Spec} H_{S}: \varepsilon_{r}-\omega \in \operatorname{Spec} H_{S}\right\} \\
& :=\left\{\varepsilon_{r} \in \operatorname{Spec} H_{S}: \exists \varepsilon_{r}^{\prime} \in \operatorname{Spec} H_{S}, \varepsilon_{r}-\varepsilon_{r}^{\prime}=\omega\right\} .
\end{aligned}
$$

With this notation the rescaled interaction Hamiltonian in its canonical form can be rewritten as

$$
\frac{1}{\lambda} H_{I}\left(t / \lambda^{2}\right):=\sum_{j} \sum_{\omega \in F} E_{\omega}^{*}\left(D_{j}\right) \int d k g_{j}^{*}(k) a_{\lambda, \omega}(t, k)+\text { h.c. },
$$

where we have introduced the operators

$$
E_{\omega}\left(D_{j}\right):=\sum_{\varepsilon_{r} \in F_{\omega}} P_{\varepsilon_{r}-\omega} D_{j} P_{\varepsilon_{r}}
$$

and the rescaled creation and annihilation densities $a_{\lambda, \omega}^{ \pm}(t, k)$ are given in Eq.(2). From Eq.(11) and Eq.(1) we obtain

$$
\begin{array}{r}
\frac{1}{\lambda^{2}}\left\langle H_{I}\left(\frac{t_{1}}{\lambda^{2}}\right) H_{I}\left(\frac{t_{2}}{\lambda^{2}}\right)\right\rangle=\frac{1}{\lambda^{2}} \sum_{i j} \sum_{\omega, \omega^{\prime} \in F}\left\{\int d k g_{i}^{*}(k) g_{j}(k) \times\right. \\
\times\left(e^{i\left(\frac{t_{2}-t_{1}}{\lambda^{2}}(\omega(k)-\omega)+\frac{t_{1}}{\lambda^{2}}\left(\omega-\omega^{\prime}\right)\right)} N(k)+1\right) E_{\omega^{\prime}}^{*}\left(D_{i}\right) E_{\omega}\left(D_{j}\right)+ \\
\left.+\int d k g_{i}(k) g_{j}^{*}(k) e^{-i\left(\frac{t_{2}-t_{1}}{\lambda^{2}}(\omega(k)-\omega)+\frac{t_{1}}{\lambda^{2}}\left(\omega-\omega^{\prime}\right)\right)} N(k) E_{\omega^{\prime}}\left(D_{i}\right) E_{\omega}^{*}\left(D_{j}\right)\right\} .
\end{array}
$$

3 The area of the unit sphere in $\mathbb{R}^{d}$ is $\int_{S_{1}} d \sigma_{S_{1}}=\frac{2 \pi^{\frac{d}{2}}}{\Gamma\left(\frac{d}{2}\right)}$. Recall that, for $d \in \mathbb{N}$, one has $\Gamma(d+1)=d$ ! and $\Gamma\left(d+\frac{1}{2}\right)=\frac{(2 d) !}{2^{2 d} d !} \sqrt{\pi}$. 
Making in Eq.(4) the change of variables $\tau=\left(t_{2}-t_{1}\right) / \lambda^{2}$ we get

$$
\begin{array}{r}
\lim _{\lambda \rightarrow 0} \sum_{i j} \sum_{\omega, \omega^{\prime} \in F}\left\{\int_{t}^{t+d t} d t_{1} e^{i \frac{t_{1}}{\lambda^{2}}\left(\omega-\omega^{\prime}\right)} \int_{\frac{t-t_{1}}{\lambda^{2}}}^{0} d \tau \int d k g_{i}^{*}(k) g_{j}(k) \times\right. \\
\times \int_{t}^{i \tau(\omega(k)-\omega)}(N(k)+1) E_{\omega^{\prime}}^{*}\left(D_{i}\right) E_{\omega}\left(D_{j}\right)+ \\
\left.\times e^{-i \tau(\omega(k)-\omega)} N(k) E_{\frac{\omega^{\prime}}{\lambda^{2}}}\left(D_{i}\right) E_{\omega}^{*}\left(D_{j}\right)\right\}
\end{array}
$$

Now the result comes from Lemmas 5 and 6 below. Some insights about the contribution of the singular region $\Gamma_{3}$ are given in Remark 7 .

LEMMA 5. Under the conditions for $\omega(k)$ given in Section 3, for every $\omega, \omega^{\prime} \in \mathbb{R}$ we have that, in $\mathcal{S}^{\prime}\left(\mathbb{R}^{d}\right),{ }^{4}$

$$
\begin{aligned}
& \lim _{\lambda \rightarrow 0} \int_{t}^{t+d t} d t_{1} e^{ \pm i \frac{t_{1}}{\lambda^{2}}\left(\omega-\omega^{\prime}\right)} \int_{\frac{t-t_{1}}{\lambda^{2}}}^{0} d \tau \chi_{\Gamma_{1}}(k) e^{ \pm i \tau(\omega(k)-\omega)}= \\
= & \delta\left(\omega-\omega^{\prime}\right) \chi_{\Gamma_{1}}(k)\left[\pi \chi_{\Gamma_{1}^{1}}(\omega) \delta(\omega(k)-\omega) \mp i P . P . \frac{1}{\omega(k)-\omega}\right],
\end{aligned}
$$

that is, for any test function $f \in \mathcal{S}\left(\mathbb{R}^{d}\right)$,

$$
\begin{gathered}
\lim _{\lambda \rightarrow 0} \int_{t}^{t+d t} d t_{1} e^{ \pm i \frac{t_{1}}{\lambda^{2}}\left(\omega-\omega^{\prime}\right)} \int_{\frac{t-t_{1}}{\lambda^{2}}}^{0} d \tau \int_{\Gamma_{1}} d k e^{ \pm i \tau(\omega(k)-\omega)} f(k)= \\
\begin{cases}\pi \chi_{\Gamma_{1}^{1}}(\omega) \int_{\Gamma_{1}} d k \delta(\omega(k)-\omega) f(k) \mp i P . P . \int_{\Gamma_{1}} d k \frac{f(k)}{\omega(k)-\omega}, & \text { if } \omega=\omega^{\prime}, \\
0, & \text { if } \omega \neq \omega^{\prime} .\end{cases}
\end{gathered}
$$

LEMMA 6. Under the conditions for $\omega(k)$ given in Section 3, suppose that the open set $\Gamma_{\alpha}$, where $\omega(k)$ is constant and equal to $\alpha$, is not empty. Then, for every $\omega, \omega^{\prime} \in \mathbb{R}$, we have that, in $\mathcal{S}^{\prime}\left(\mathbb{R}^{d}\right)$,

$$
\lim _{\lambda \rightarrow 0} \int_{t}^{t+d t} d t_{1} e^{ \pm i \frac{t_{1}}{\lambda^{2}}\left(\omega-\omega^{\prime}\right)} \int_{\frac{t-t_{1}}{\lambda^{2}}}^{0} d \tau \chi_{\Gamma_{\alpha}}(k) e^{ \pm i \tau(\omega(k)-\omega)}=0,
$$

that is, for any test function $f \in \mathcal{S}\left(\mathbb{R}^{d}\right)$,

$$
\lim _{\lambda \rightarrow 0} \int_{t}^{t+d t} d t_{1} e^{ \pm i \frac{t_{1}}{\lambda^{2}}\left(\omega-\omega^{\prime}\right)} \int_{\frac{t-t_{1}}{\lambda^{2}}}^{0} d \tau \int_{\Gamma_{\alpha}} d k e^{ \pm i \tau(\omega(k)-\omega)} f(k)=0 .
$$

${ }^{4}$ These formulas are correct whether if we consider a bilinear dual pair $\langle\cdot, \cdot\rangle$, i.e. $\langle f, g\rangle=\int f g$, or if the dual pair $\langle\cdot, \cdot\rangle$ is antilinear on the left and linear on the right, i.e. $\langle f, g\rangle=\int f^{*} g$. 
REMARK 7. Under the conditions for $\omega(k)$ given in Section 3, assume that $\Gamma_{3}$ is a $d-1$ dimensional regular surface which is the boundary of two open regions $\Gamma_{\alpha_{1}}$ and $\Gamma_{\alpha_{2}}$ (subsets of $\Gamma_{2}$ ) and such that

$$
\omega(k)=\beta, \quad \forall k \in \Gamma_{3} .
$$

For each $\epsilon>0$ consider an open region $\Gamma_{\epsilon} \subset \mathbb{R}^{d}$ such that

(i) $\Gamma_{3} \subset \Gamma_{\epsilon_{1}} \subset \Gamma_{\epsilon_{2}}$ if $\epsilon_{1}<\epsilon_{2}$,

(ii) $\Gamma_{3}=\cap_{\epsilon>0} \Gamma_{\epsilon}$,

and, on each $\Gamma_{\epsilon}$, replace $\omega(k)$ by a regular function $\omega_{\epsilon}(k)$ in such a way that, in some sense,

$$
\lim _{\epsilon \rightarrow 0} \omega_{\epsilon}=\omega .
$$

Now, for $\omega, \omega^{\prime} \in \mathbb{R}$, let us study the following limit in $\mathcal{S}^{\prime}\left(\mathbb{R}^{d}\right)$ :

$$
F:=\lim _{\epsilon \rightarrow 0} \lim _{\lambda \rightarrow 0} \int_{t}^{t+d t} d t_{1} e^{ \pm i \frac{t_{1}}{\lambda^{2}}\left(\omega-\omega^{\prime}\right)} \int_{\frac{t-t_{1}}{\lambda^{2}}}^{0} d \tau \chi_{\Gamma_{\epsilon}}(k) e^{ \pm i \tau\left(\omega_{\epsilon}(k)-\omega\right)} .
$$

Clearly, if our choice is $\omega_{\epsilon}(k)=c$ for every $k \in \Gamma_{\epsilon}$ and $\epsilon>0$, then, by Proposition $6, F=0$ or $F$ doesn't exist. On the other hand, if the functions $\omega_{\epsilon}$ are such that we can apply Proposition 5 on $\Gamma_{\epsilon}$, then

$$
F=\delta\left(\omega-\omega^{\prime}\right) \lim _{\epsilon \rightarrow 0} \chi_{\Gamma_{\epsilon}}(k)\left[\pi \chi_{\Gamma_{\epsilon}^{1}}(\omega) \delta\left(\omega_{\epsilon}(k)-\omega\right) \mp i \text { P.P. } \frac{1}{\omega_{\epsilon}(k)-\omega}\right],
$$

but this limit depends on the form of the functions $\omega_{\epsilon}$. In particular, the limit depends on $\Gamma_{\epsilon}^{1}:=\operatorname{Rang}\left(\left.\omega_{\epsilon}\right|_{\Gamma_{\epsilon}}\right)$.

Proof of Lemma 5: The factor $\delta\left(\omega-\omega^{\prime}\right)$ comes from

$$
\begin{aligned}
\lim _{\lambda \rightarrow 0} \int_{t}^{t+d t} d t_{1} e^{ \pm i \frac{t_{1}}{\lambda^{2}}\left(\omega-\omega^{\prime}\right)}= \\
= \begin{cases}\left.t_{1}\right|_{t_{1}=t} ^{t_{1}=t+d t}=d t, & \text { if } \omega=\omega^{\prime}, \\
\left.\lim _{\lambda \rightarrow 0} \lambda^{2} \frac{e^{ \pm i \frac{t_{1}}{\lambda^{2}}\left(\omega-\omega^{\prime}\right)}}{ \pm i\left(\omega-\omega^{\prime}\right)}\right|_{t_{1}=t} ^{t_{1}=t+d t}=0, & \text { if } \omega \neq \omega^{\prime},\end{cases}
\end{aligned}
$$

since

$$
\begin{aligned}
& \left.\lim _{\lambda \rightarrow 0} \lambda^{2} \frac{e^{ \pm i \frac{t_{1}}{\lambda^{2}}\left(\omega-\omega^{\prime}\right)}}{ \pm i\left(\omega-\omega^{\prime}\right)}\right|_{t_{1}=t} ^{t_{1}=t+d t}= \\
& =\lim _{\lambda \rightarrow 0} e^{ \pm i \frac{t}{\lambda^{2}}\left(\omega-\omega^{\prime}\right)} \frac{\lambda^{2}}{ \pm i\left(\omega-\omega^{\prime}\right)}\left(e^{ \pm i \frac{d t}{\lambda^{2}}\left(\omega-\omega^{\prime}\right)}-1\right)= \\
& =\left.\lim _{\lambda \rightarrow 0} e^{ \pm i \frac{t}{\lambda^{2}}\left(\omega-\omega^{\prime}\right)} \frac{\lambda^{2}}{ \pm i\left(\omega-\omega^{\prime}\right)} \frac{d}{d t} e^{ \pm i \frac{t}{\lambda^{2}}\left(\omega-\omega^{\prime}\right)}\right|_{t=0} d t= \\
& =\lim _{\lambda \rightarrow 0} e^{ \pm i \frac{t}{\lambda^{2}}\left(\omega-\omega^{\prime}\right)} d t=0 \quad \text { (by Lemma 8). }
\end{aligned}
$$


Using the notation given in Section 3.(i), we have

$$
\begin{gathered}
\int_{-\infty}^{0} d \tau \int_{\Gamma_{1}} d k e^{i \tau(\omega(k)-\omega)} f(k)=\int_{-\infty}^{0} d \tau \int_{\Gamma_{1}^{1}} d u_{1} e^{i \tau\left(u_{1}-\omega\right)} \Omega_{f}\left(u_{1}\right)= \\
=\int d \tau e^{-i \tau \omega} \theta(-\tau) \int d u_{1} e^{i \tau u_{1}} \chi_{\Gamma_{1}^{1}}\left(u_{1}\right) \Omega_{f}\left(u_{1}\right)
\end{gathered}
$$

where $\theta$ is the Heaviside function. To analyze the last expression assume that $\chi_{\Gamma_{1}^{1}}\left(u_{1}\right) \Omega_{f}\left(u_{1}\right)$ is of bounded support on $u_{1}$, else we can consider a convenient partition of the unity for $\Gamma_{1}$. Then $\chi_{\Gamma_{1}^{1}}\left(u_{1}\right) \Omega_{f}\left(u_{1}\right)$ is convolutible with any other tempered distribution and its Fourier transform is a $C^{\infty}$ function, so that the product $\theta(-\tau)\left[\chi_{\Gamma_{1}^{1}} \Omega_{f}\right]^{\wedge}(\tau)$ is also well defined in $\mathcal{S}^{\prime}(\mathbb{R})$. Thus we can write

$$
\begin{aligned}
& \int d \tau e^{-i \tau \omega} \theta(-\tau) \int d u_{1} e^{i \tau u_{1}} \chi_{\Gamma_{1}^{1}}\left(u_{1}\right) \Omega_{f}\left(u_{1}\right)= \\
& =\int d \tau e^{-i \tau \omega} \theta(-\tau)\left[\chi_{\Gamma_{1}^{1}} \Omega_{f}\right]^{\wedge}(\tau)=\sqrt{2 \pi}\left[\theta(-\tau)\left[\chi_{\Gamma_{1}^{1}} \Omega_{f}\right]^{\wedge}(\tau)\right]^{\vee}(\omega)= \\
& \left.=\sqrt{2 \pi}\left([\theta(-\tau)]^{\vee}\left(u_{1}\right) *\left[\left[\chi_{\Gamma_{1}^{1}} \Omega_{f}\right]^{\wedge}(\tau)\right]^{\vee}\left(u_{1}\right)\right]\right)(\omega)= \\
& \left.\left.=\sqrt{2 \pi} \frac{1}{\sqrt{2 \pi}}\left(\pi \delta\left(u_{1}\right)+i \text { P.P. } \frac{1}{u_{1}}\right) *\left[\chi_{\Gamma_{1}^{1}} \Omega_{f}\right]\left(u_{1}\right)\right]\right)(\omega)= \\
& =\int d u_{1}\left(\pi \delta\left(\omega-u_{1}\right)+i \text { P.P. } \frac{1}{\omega-u_{1}}\right)\left[\chi_{\Gamma_{1}^{1}} \Omega_{f}\right]\left(u_{1}\right)= \\
& =\pi \chi_{\Gamma_{1}^{1}}(\omega) \Omega_{f}(\omega)+i \text { P.P. } \int d u_{1} \frac{1}{\omega-u_{1}} \chi_{\Gamma_{1}^{1}}\left(u_{1}\right) \Omega_{f}\left(u_{1}\right)= \\
& =\pi \chi_{\Gamma_{1}^{1}}(\omega) \int_{S_{u_{1}=\omega}} d \sigma_{S_{u_{1}}=\omega} f(\psi(u))- \\
& \quad-i \mathrm{P} . \mathrm{P} . \int_{\Gamma_{1}^{1}} d u_{1} \frac{1}{u_{1}-\omega} \int_{S_{u_{1}}} d \sigma_{S_{u_{1}}} f(\psi(u))= \\
& =\pi \chi_{\Gamma_{1}^{1}}(\omega)\langle\delta(\omega(k)-\omega), f\rangle-i \text { P.P. } \int_{\Gamma_{1}} d k \frac{f(k)}{\omega(k)-\omega}= \\
& =\pi \chi_{\Gamma_{1}^{1}}(\omega)\langle\delta(\omega(k)-\omega), f\rangle+i\left\langle\text { P.P. } \frac{\chi_{\Gamma_{1}}(k)}{\omega(k)-\omega}, f(k)\right\rangle .
\end{aligned}
$$

This concludes the proof. 
Proof of Lemma 6: Consider any of the open sets $\Gamma_{\alpha}$, where $\omega(k)$ is constant and equal to $\alpha$. There we have

$$
\begin{aligned}
& \lim _{\lambda \rightarrow 0} \int_{t}^{t+d t} d t_{1} e^{ \pm i \frac{t_{1}}{\lambda^{2}}\left(\omega-\omega^{\prime}\right)} \int_{\frac{t-t_{1}}{\lambda^{2}}}^{0} d \tau \chi_{\Gamma_{\alpha}}(k) e^{ \pm i \tau(\omega(k)-\omega)}= \\
& =\chi_{\Gamma_{\alpha}}(k) \lim _{\lambda \rightarrow 0} \int_{t}^{t+d t} d t_{1} e^{ \pm i \frac{t_{1}}{\lambda^{2}}\left(\omega-\omega^{\prime}\right)} \int_{\frac{t-t_{1}}{\lambda^{2}}}^{0} d \tau e^{ \pm i \tau(\alpha-\omega)}= \\
& =\left\{\begin{array}{l}
\chi_{\Gamma_{\alpha}}(k) \lim _{\lambda \rightarrow 0} \int_{t}^{t+d t} d t_{1} e^{ \pm i \frac{t_{1}}{\lambda^{2}}\left(\omega-\omega^{\prime}\right)} \frac{-t+t_{1}}{\lambda^{2}}, \quad \text { for } \alpha=\omega ; \\
\chi_{\Gamma_{\alpha}}(k) \lim _{\lambda \rightarrow 0} \int_{t}^{t+d t} d t_{1} e^{ \pm i \frac{t_{1}}{\lambda^{2}}\left(\omega-\omega^{\prime}\right)} \frac{1}{ \pm i(\alpha-\omega)}\left(1-e^{ \pm i \frac{t-t_{1}}{\lambda^{2}}(\alpha-\omega)}\right), \\
\text { for } \alpha \neq \omega .
\end{array}\right.
\end{aligned}
$$

For $\omega=\omega^{\prime}$ we have, if $\alpha=\omega$,

$$
\lim _{\lambda \rightarrow 0} \int_{t}^{t+d t} d t_{1} \frac{-t+t_{1}}{\lambda^{2}}=\lim _{\lambda \rightarrow 0} \frac{(d t)^{2}}{2 \lambda^{2}}=0 \quad\left(\text { since }(d t)^{2}=0\right)
$$

and, if $\alpha \neq \omega$,

$$
\begin{aligned}
& \frac{1}{ \pm i(\alpha-\omega)} \lim _{\lambda \rightarrow 0} \int_{t}^{t+d t} d t_{1}\left(1-e^{ \pm i \frac{t-t_{1}}{\lambda^{2}}(\alpha-\omega)}\right)= \\
& =\frac{1}{ \pm i(\alpha-\omega)} \lim _{\lambda \rightarrow 0}\left(d t-\left.\frac{\lambda^{2}}{\mp i(\alpha-\omega)} e^{ \pm i \frac{t}{\lambda^{2}}(\alpha-\omega)} e^{\mp i \frac{t_{1}}{\lambda^{2}}(\alpha-\omega)}\right|_{t_{1}=t} ^{t_{1}=t+d t}\right)= \\
& =\frac{1}{ \pm i(\alpha-\omega)} \lim _{\lambda \rightarrow 0}\left(d t-\frac{\lambda^{2}}{\mp i(\alpha-\omega)}\left(e^{\mp i \frac{d t}{\lambda^{2}}(\alpha-\omega)}-1\right)\right)= \\
& =\frac{1}{ \pm i(\alpha-\omega)} \lim _{\lambda \rightarrow 0}\left(1-\left.\frac{\lambda^{2}}{\mp i(\alpha-\omega)} \frac{d}{d t} e^{\mp i \frac{t}{\lambda^{2}}(\alpha-\omega)}\right|_{t=0}\right) d t= \\
& =\frac{1}{ \pm i(\alpha-\omega)} \lim _{\lambda \rightarrow 0}(1-1) d t=0 .
\end{aligned}
$$

For $\omega \neq \omega^{\prime}$ we have, if $\alpha=\omega$,

$$
\begin{gathered}
\lim _{\lambda \rightarrow 0} \int_{t}^{t+d t} d t_{1} e^{ \pm i \frac{t_{1}}{\lambda^{2}}\left(\omega-\omega^{\prime}\right)} \frac{-t+t_{1}}{\lambda^{2}}= \\
=\lim _{\lambda \rightarrow 0}\left\{\left(-\frac{t}{\lambda^{2}}-\frac{1}{ \pm i\left(\omega-\omega^{\prime}\right)}\right) \int_{t}^{t+d t} d t_{1} e^{ \pm i \frac{t_{1}}{\lambda^{2}}\left(\omega-\omega^{\prime}\right)}+\right. \\
\left.\quad+\left.\frac{t_{1} e^{ \pm i \frac{t_{1}}{\lambda^{2}}\left(\omega-\omega^{\prime}\right)}}{ \pm i\left(\omega-\omega^{\prime}\right)}\right|_{t_{1}=t} ^{t_{1}=t+d t}\right\}= \\
=\lim _{\lambda \rightarrow 0}\left\{\left(-\frac{t}{\lambda^{2}}-\frac{1}{ \pm i\left(\omega-\omega^{\prime}\right)}\right) e^{ \pm i \frac{t}{\lambda^{2}}\left(\omega-\omega^{\prime}\right)} d t+\right. \\
\left.\quad+\frac{e^{ \pm i \frac{t}{\lambda^{2}}\left(\omega-\omega^{\prime}\right)}}{ \pm i\left(\omega-\omega^{\prime}\right)}\left(1+\frac{\lambda^{2}}{ \pm i\left(\omega-\omega^{\prime}\right)}(t+d t)\right) d t\right\}= \\
=\lim _{\lambda \rightarrow 0} \frac{-t}{\lambda^{2}} e^{ \pm i \frac{t}{\lambda^{2}}\left(\omega-\omega^{\prime}\right)} d t=0 \quad(\text { by Lemma 8) }
\end{gathered}
$$


and, if $\alpha \neq \omega$,

$$
\begin{aligned}
& \lim _{\lambda \rightarrow 0} \int_{t}^{t+d t} d t_{1} e^{ \pm i \frac{t_{1}}{\lambda^{2}}\left(\omega-\omega^{\prime}\right)} \frac{1}{ \pm i(\alpha-\omega)}\left(1-e^{ \pm i \frac{t-t_{1}}{\lambda^{2}}(\alpha-\omega)}\right)=\frac{1}{ \pm i(\alpha-\omega)} \times \\
& \quad \times \lim _{\lambda \rightarrow 0}\left\{e^{ \pm i \frac{t}{\lambda^{2}}\left(\omega-\omega^{\prime}\right)} d t-e^{ \pm i \frac{t}{\lambda^{2}}(\alpha-\omega)} \int_{t}^{t+d t} d t_{1} e^{ \pm i \frac{t_{1}}{\lambda^{2}}\left(2 \omega-\omega^{\prime}-\alpha\right)}\right\}= \\
& =\frac{1}{ \pm i(\alpha-\omega)} \lim _{\lambda \rightarrow 0}\left\{e^{ \pm i \frac{t}{\lambda^{2}}\left(\omega-\omega^{\prime}\right)} d t-e^{ \pm i \frac{t}{\lambda^{2}}(\alpha-\omega)} e^{ \pm i \frac{t}{\lambda^{2}}\left(2 \omega-\omega^{\prime}-\alpha\right)} d t\right\}=0 .
\end{aligned}
$$

This concludes the proof.

\section{Appendix}

The following appendices include some of the conventions and results used in this paper about the Fourier transform in Schwartz spaces and the ordinary convergence of Fourier integrals.

\section{A. Fourier Transforms}

We shall use the following conventions: ${ }^{5}$ The dual pair $\langle\cdot, \cdot\rangle$ is antilinear on the left and linear on the right, i.e. $\langle f, g\rangle=\int f^{*} g$, where * denotes the complex conjugate. The Fourier transform $f^{\wedge}$ and the inverse Fourier transform $f^{\vee}$ of a test function $f \in \mathcal{S}\left(\mathbb{R}^{d}\right)$ are given by

$$
f^{\wedge}(s):=\frac{1}{(2 \pi)^{d / 2}} \int d x f(x) e^{i x \cdot s}, \quad f^{\vee}(s):=\frac{1}{(2 \pi)^{d / 2}} \int d x f(x) e^{-i x \cdot s},
$$

so that $f^{\wedge \vee}=f^{\vee \wedge}=f$. The Fourier transform $F^{\wedge}$ and the inverse Fourier transform $F^{\vee}$ of a distribution $F \in \mathcal{S}^{\prime}\left(\mathbb{R}^{d}\right)$ are defined by the relations

$$
\left\langle F^{\wedge}, f^{\wedge}\right\rangle=\langle F, f\rangle, \quad\left\langle F^{\vee}, f^{\vee}\right\rangle=\langle F, f\rangle,
$$

so that, if $F \in L^{1}$, then

$$
\begin{gathered}
F^{\wedge}(s)=\frac{1}{(2 \pi)^{d / 2}}\left\langle F^{*}(x), e^{i x \cdot s}\right\rangle=\frac{1}{(2 \pi)^{d / 2}} \int d x F(x) e^{i x \cdot s}, \\
F^{\vee}(s)=\frac{1}{(2 \pi)^{d / 2}}\left\langle F^{*}(x), e^{-i x \cdot s}\right\rangle=\frac{1}{(2 \pi)^{d / 2}} \int d x F(x) e^{-i x \cdot s} .
\end{gathered}
$$

5 These conventions are the Gelfand-Shilov's ones [3] except by the factors $(2 \pi)^{-d / 2}$ in the Fourier transform and its inverse. Our (inverse) Fourier transforms, in $\mathcal{S}\left(\mathbb{R}^{d}\right)$ and $\mathcal{S}^{\prime}\left(\mathbb{R}^{d}\right)$, are obtained from the Gelfand-Shilov (inverse) Fourier transforms (multiplying) dividing by $(2 \pi)^{d / 2}$. 
Thus,

$$
\begin{gathered}
\int_{-\infty}^{0} e^{i t \omega} d t=(2 \pi)^{d / 2}[\theta(-t)]^{\wedge}(\omega)=\frac{-i}{\omega-i 0}=\pi \delta(\omega)-i \text { P.P. } \frac{1}{\omega} \\
\int_{-\infty}^{0} e^{-i t \omega} d t=(2 \pi)^{d / 2}[\theta(-t)]^{\wedge}(\omega)^{*}=\frac{i}{\omega+i 0}=\pi \delta(\omega)+i \text { P.P. } \frac{1}{\omega} .
\end{gathered}
$$

We shall need the following result:

LEMMA 8. For every $n \in \mathbb{N}$, and $\omega \neq 0$ we have

$$
\lim _{\lambda \rightarrow 0}\left(\frac{t}{\lambda^{2}}\right)^{n} e^{ \pm i \frac{t}{\lambda^{2}} \omega}=0 \quad \text { in } \mathcal{S}^{\prime}(\mathbb{R}) .
$$

Proof: Remember that for every $m \in \mathbb{N}$ there exist a constant $C_{m}>0$ such that for any test function $\varphi \in \mathcal{S}(\mathbb{R})$, every $n \in \mathbb{N}$ and $\omega \neq 0$ we have (see section V.1.3 of Schwartz [4])

$$
\left|\left\langle t^{n} e^{ \pm i t \omega}, \varphi(t)\right\rangle\right|=\left|\int d t e^{\mp i t \omega} t^{n} \varphi(t)\right| \leq C_{m} \frac{\left\|\frac{d^{m}}{d t^{m}}\left[t^{n} \varphi(t)\right]\right\|_{L^{1}}}{|\omega|^{m}} .
$$

Then, taking $m>n$, for every $\omega \neq 0$ we get

$$
\lim _{\lambda \rightarrow 0}\left|\left\langle\left(\frac{t}{\lambda^{2}}\right)^{n} e^{ \pm i \frac{t}{\lambda^{2}} \omega}, \varphi(t)\right\rangle\right| \leq \lim _{\lambda \rightarrow 0} C_{m} \lambda^{2(m-n)} \frac{\left\|\frac{d^{m}}{d t^{m}}\left[t^{n} \varphi(t)\right]\right\|_{L^{1}}}{|\omega|^{m}}=0 .
$$

This implies the result.

\section{B. Summability of Fourier Integrals}

The tests of Jordan and Dini for ordinary convergence of Fourier integrals can be found in $[5$, Ths. $3,4,23]$.

THEOREM 9 (Jordan's Test). Let $f \in L^{1}(\mathbb{R})$. If $f$ is of bounded variation in an interval $(a, b)$ including $x$, then

$$
\begin{aligned}
\frac{f(x+0)+f(x-0)}{2} & =\frac{1}{\pi} \lim _{\lambda \rightarrow \infty} \int_{0}^{\lambda} d u \int_{-\infty}^{\infty} f(t) \cos (u(x-t)) d t \\
& =\frac{1}{2 \pi} \lim _{\lambda \rightarrow \infty} \int_{-\lambda}^{\lambda} e^{-i x u} d u \int_{-\infty}^{\infty} f(t) e^{i x u} d t
\end{aligned}
$$

the integral converging uniformly in any interval interior to $(a, b)$. 
THEOREM 10 (Dini's Test). Let $f \in L^{1}(\mathbb{R})$. Then, for a given $x$,

$$
\begin{aligned}
f(x) & =\frac{1}{\pi} \lim _{\lambda \rightarrow \infty} \int_{0}^{\lambda} d u \int_{-\infty}^{\infty} f(t) \cos (u(x-t)) d t \\
& =\frac{1}{2 \pi} \lim _{\lambda \rightarrow \infty} \int_{-\lambda}^{\lambda} e^{-i x u} d u \int_{-\infty}^{\infty} f(t) e^{i x u} d t,
\end{aligned}
$$

is true if

$$
\int_{0}^{\delta}\left|\frac{f(x+y)+f(x-y)-2 f(x)}{y}\right| d y
$$

exists for some positive $\delta$; in particular it holds if $f$ is differentiable at the point $x$.

\section{References}

1. Accardi L., Lu Y.G. and Volovich I., Quantum Theory and Its Stochastic Limit, Springer-Verlag, Berlin, 2002.

2. Accardi L. and Kozyrev S.V., Quantum Interacting Particle Systems, in L. Accardi and F. Fagnola (eds.), Quantum Interacting Particle Systems, World Scientific, Singapore, 2002, 1-193.

3. Gelfand I.M. and Shilov G.E., Les Distributions, Dunod, Paris, 1962.

4. Schwartz L., Méthodes Mathématiques pour les Sciences Physiques, Hermann, Paris, 1966.

5. Titchmarsh E.C., Introduction to the Theory of Fourier Integrals, Oxford University Press, Oxford, 1948. 